\title{
Simulating the Clamped Tapered Beam Specimen under Quasi-Static and Fatigue Loading using Floating Node Method
}

\author{
B.R. Seshadri ${ }^{1}$ and N.V. de Carvalho ${ }^{1}$ \\ ${ }^{I}$ National Institute of Aerospace, resident at Durability, Damage Tolerance, and Reliability Branch, \\ NASA Langley Research Center, Hampton, VA, 23681, USA \\ J.G. Ratcliffe ${ }^{2}$ \\ ${ }^{2}$ Durability, Damage Tolerance, and Reliability Branch, \\ NASA Langley Research Center, Hampton, VA, 23681, USA \\ Gerald E. Mabson ${ }^{3}$ and Lyle Deobald ${ }^{3}$ \\ ${ }^{3}$ The Boeing Company, Seattle, WA, 98124, USA
}

\begin{abstract}
As part of the NASA Advanced Composites Project (ACP), a sub-element has been designed to provide validation data for progressive damage analysis models. The clamped tapered beam is a cross-ply laminated composite specimen designed to validate the simulation of the onset of matrix cracks and their interaction with delaminations, including delamination migration. A tapered geometry was used to localize the first damage occurrence in the tapered region, without prescribing an initial crack. The boundary and loading conditions were chosen to favor delamination growth and subsequent migration after the first damage occurrence. The typical sequence of events consists of a matrix crack located at the tapered region, leading to delamination onset, followed by delamination growth and subsequent delamination migration to a different interface via a dominant matrix crack. The Clamped Tapered Beam (CTB) was tested in both quasi-static and fatigue regimes. The results obtained are used in this study to assess and validate a methodology based on the Floating Node Method (FNM) implemented as an Extended Interface Element. In this methodology, quasi-static and fatigue damage formation and development are modeled by combining FNM to represent crack networks, with Directional Cohesive Zone Elements (DCZE) and Virtual Crack Closure Technique (VCCT), respectively. Qualitatively, the methodology is capable of predicting the sequence of events and overall failure morphology. Quantitatively, the simulation results generally bound the experimental data, based on the range of the characterization data used. In this paper, the results from quasi static and fatigue simulations are compared and correlated with experimental data.
\end{abstract}

\section{Nomenclature}

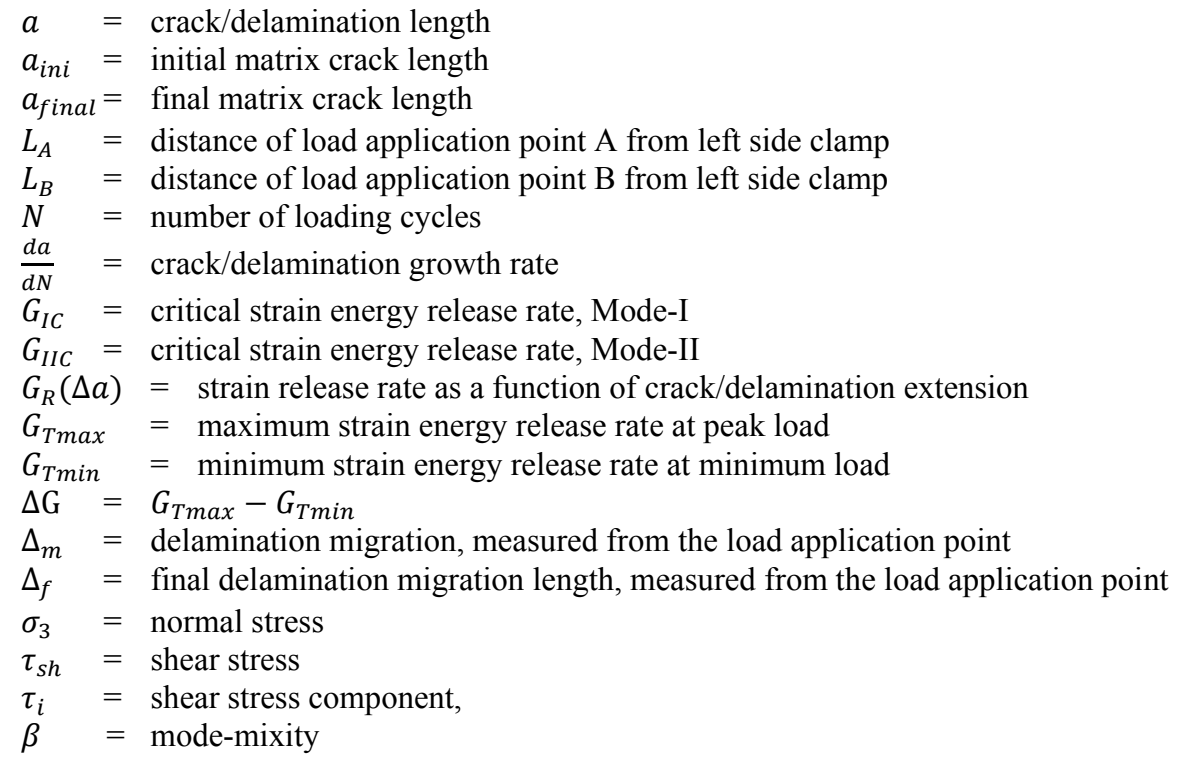




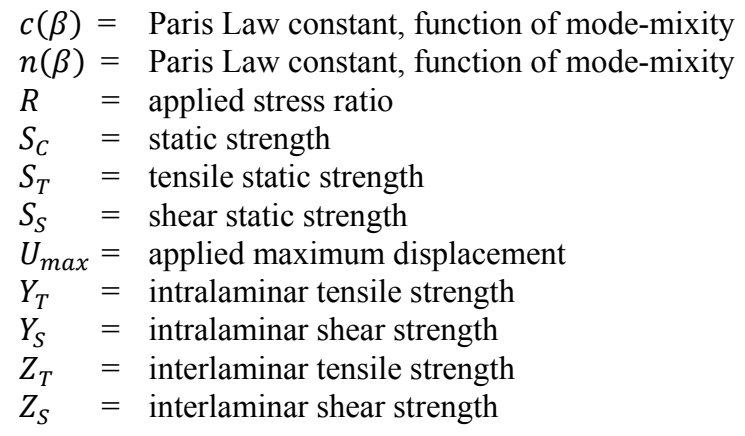

\section{Introduction}

As part of the NASA Advanced Composites Project (ACP), the clamped tapered beam specimen, illustrated in Figure 1, has been designed to provide validation data for progressive damage analysis models [1]. The present work reports on the blind predictions performed with a 3D discrete crack approach based on the Floating Node Method (FNM) [2]. The clamped tapered beam specimen was based on the delamination migration test proposed in [3-4]. The tapered geometry was devised to localize the first damage occurrence in the tapered region, without the need to prescribe an initial crack. The typical sequence of events consists of the onset of a matrix crack within the tapered region, which triggers delamination followed by subsequent delamination migration to a different interface via a dominant matrix crack.

In the present work, a methodology based on the FNM is assessed. The FNM represents discrete crack networks by generating sub-elements within cracked finite elements through the use of floating nodes [2]. No a priori location for damage initiation was assumed. The methodology does not limit the number of possible matrix cracks, delaminations or the crack spacing, thereby accommodating the initiation and propagation of complex crack networks at the meso-scale, without the need for re-meshing.

To model quasi-static delamination and matrix crack formation and growth, the FNM strategy was combined with Directional Cohesive Zone Elements (DCZE) [5]. To model fatigue damage formation and growth, the FNM approach was combined with an element-based Virtual Crack Closure Technique (VCCT) to model growth of delaminations and matrix cracks, and a stress criterion to model their initiation [6]. To facilitate this behavior, the concept of a propagation zone [6] was used to combine Linear Elastic Fracture Mechanics (LEFM) based VCCT approach for growth along with a stress based criterion to model crack initiation. The process zone was defined [6] ahead of the crack tip where the onset criterion was not activated. To carry out blind prediction simulations, the geometric boundary and loading conditions used in the experiments were made known, in order to get the correct global response. The experimental details, such as location of delamination initiation, growth, migration and loaddisplacement data from static testing, and delamination length versus number of cycles under fatigue loading, were not shared before the simulation. The correlation with experimental data was performed after completing the blind-prediction. This helped to validate and quantify the methodology developed for the simulation purpose. The present paper presents a brief summary of experiments and methodology developed. The discussion focuses on novel aspects of the methodology, correlation of results with experimental data, and outstanding challenges.

\section{Experiments}

The clamped tapered beam specimen geometry is illustrated in Figure 1. It consists of a cross-ply composite beam, clamped on both ends and loaded via a hinge connected to a loading rod, located at a distance $L_{A}$ or $L_{B}$ from the lefthand-side clamp. The boundary and loading conditions were chosen to favor delamination growth and subsequent migration after the first damage occurrence. Varying the load-offset application point from $L_{A}$ to $L_{B}$ affects both the peak load and the migration location. The specimen was designed to ensure that the fatigue crack growth would reside in the Paris Law crack growth regime of IM7/8552 Carbon Fiber Reinforced Plastic (CFRP) for an extended length of damage growth under displacement control, thus avoiding premature damage arrestment or unstable failure. Further details on the specimen and design considerations are given in [1]. 


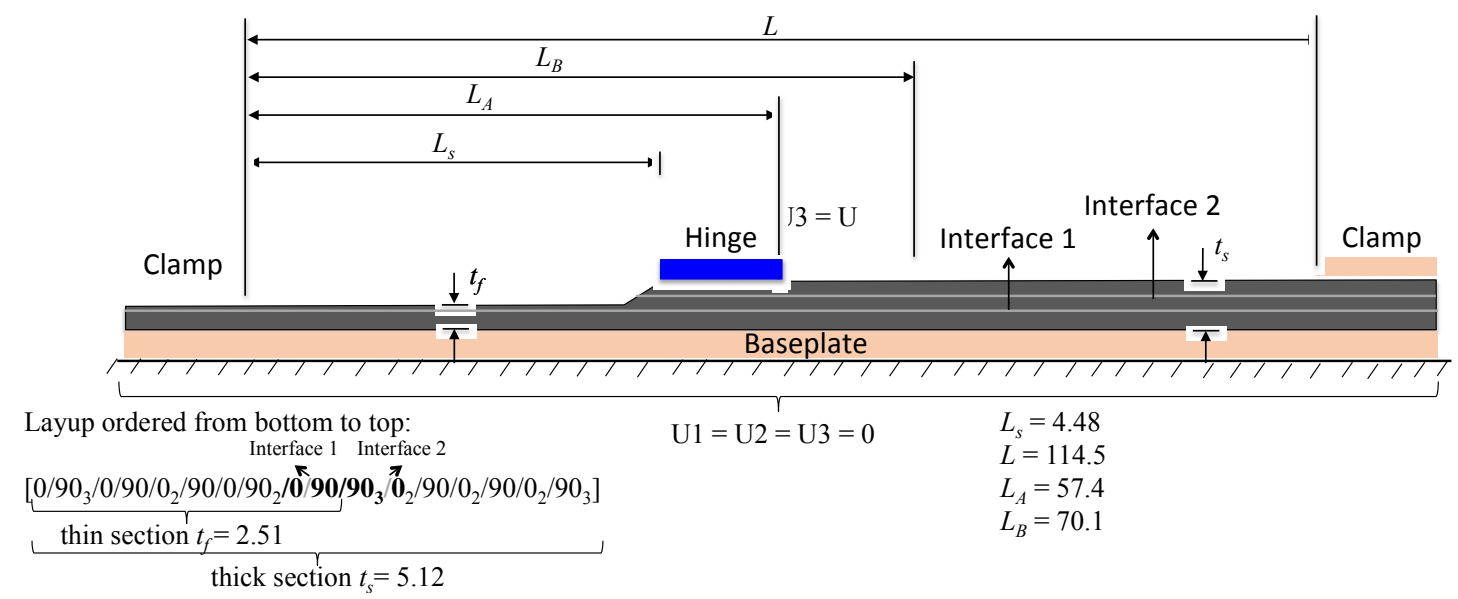

Fig. 1 Clamped tapered beam specimen dimensions (not to scale).

A typical scenario of failure events observed during experiment is depicted in Figure 2. Upon a critical amount of loading, a matrix crack forms at location (1) and grows toward location (2) at "interface 1" where a delamination is initiated. With further loading, delamination grows from location (2) toward (3) after which migration occurs via a matrix crack through the stack of $90^{\circ}$ plies, initiating a delamination at "interface 2 " (4). After the occurrence of migration, with further continuation of loading, the delamination grows from location (4) to (5). The distance $\Delta_{M}$ is measured from the load application point indicating the beginning migration of delamination.

Two sets of displacement controlled quasi-static tests were performed by applying displacement at locations $L_{A}$ or $L_{B}$, as depicted in Figure 1. Under fatigue loading, the offset chosen was $L_{A}$. Two series of fatigue tests were performed with different maximum applied displacements: $U_{\max }=0.91 \mathrm{~mm}$ (high) and $U_{\max }=0.825 \mathrm{~mm}$ (low). Both cases were tested with an R-ratio $\left(\mathrm{U}_{\min } / \mathrm{U}_{\max }\right)$ of $\mathrm{R}=0.2$, and frequency of $5 \mathrm{~Hz}$. Load-displacement, migration location $(\Delta \mathrm{m})$, and delamination length as a function of cycle count were measured correlated with the results obtained from the simulations.

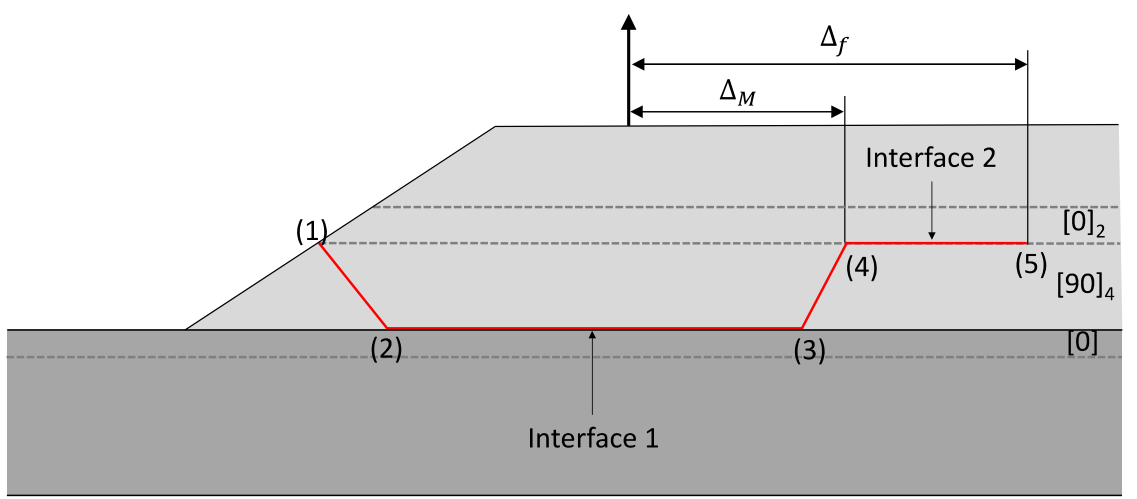

Fig. 2 Typical failure scenario and events selected for correlation with experimental data (not to scale).

\section{Methodology}

The methodology used in the blind-predictions exercise under quasi-static and fatigue loading is outlined. Overall, the methodology is based on [7] for quasi-static and [6,8] for fatigue. with emphasis on the physical models used and novel features. 


\section{A. Floating Node Method extended interface element}

The extended interface element consists of a 3D 48 noded element, comprised of 16 real nodes which have preassigned positions, and 32 floating nodes as shown in Figure 3a. The real nodes are used to define two sub-elements: one above and another below an interface element. The 32 floating nodes are the minimum subset of floating nodes that enable the two sub-elements to split independently. The floating nodes have their topological relationships to the element assigned as regular nodes, being part of the element connectivity list. However, if the element is not split, their degrees of freedom are not activated, and they can be removed from the system of equations. If the sub-elements need to be split to accommodate matrix cracks, the floating nodes were used as required. Since the connectivity of the floating nodes was defined 'a priori', when activated they naturally enforce crack path continuity. For example, an edge was split by a unique pair of floating nodes shared by all the elements sharing that edge, as defined by their connectivity list. The element can be used to represent matrix cracks of any in-plane orientation. As matrix cracks nucleate as shown in Figure 3b, the interface element may be also split using floating nodes, and interface elements were assigned as required to model the subsequent opening of the newly generated splits.

As depicted in Figure 3b, the matrix cracks were assumed to be straight through-thickness. This approximation was used to facilitate the integration of partitioned elements. However, matrix cracks will only be straight throughthickness if forming under predominantly tensile loads, otherwise, their angle may vary. In order to mitigate the effect of this approximation, the present study used two corrections. The first consists in determining the tractions in a rotated coordinate system, following $[9,10]$. In addition, since the matrix cracks were free to follow a mode I path as they propagate through-thickness, it was also considered that the shear component determined along direction 1 in Figure 4 , if not zero, does not contribute to the mode-mixity.

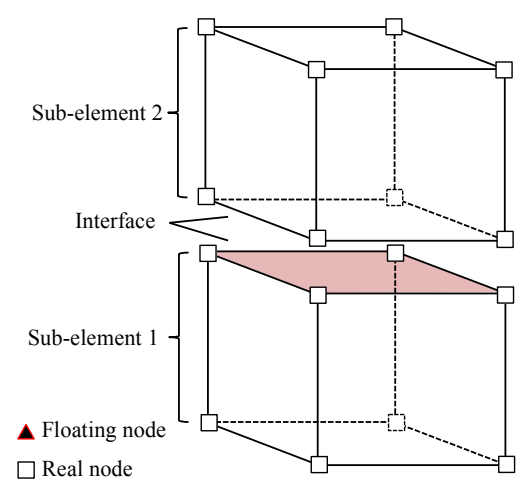

(a) Pristine extended interface element with sub-elements above and below an interface

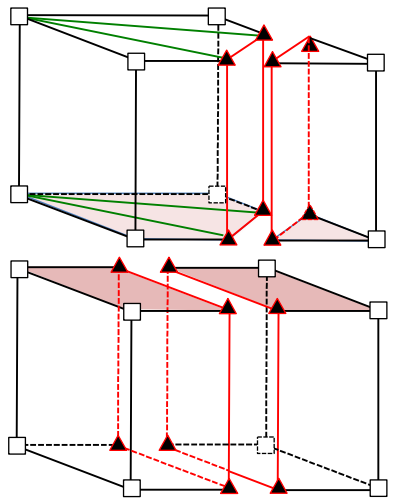

(b) element representing matrix cracks

Fig. 3 Extended interface element. Distances between bettween sub-elements and partitions are exagerated for illustration purposes.

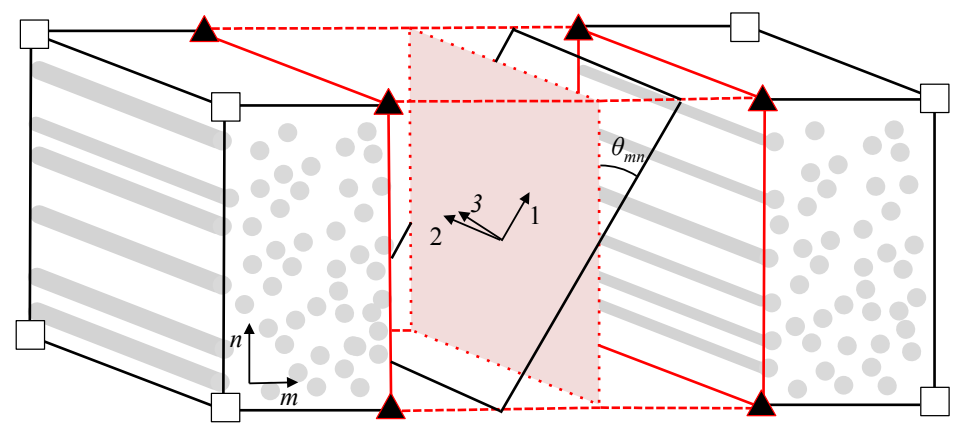

Fig. 4 Matrix crack through thickness approximation. 


\section{B. Delamination and Matrix cracks: onset and propagation}

\section{Static}

Delamination onset and propagation under quasi-static loading conditions were modeled using Directional Cohesive Zone Elements pre-assigned at the interface between sub-elements 1 and 2. The cohesive elements constitutive response follows [11]. In this formulation, unloading initiates as determined by a BK criterion written in stress space, when the effective traction $\tau_{e}$

$$
\left(\tau_{e}\right)^{2}=\left(\left\langle\sigma_{3}\right\rangle\right)^{2}+\left(\tau_{s h}\right)^{2} \beta^{\eta}
$$

exceeds the strength, given by:

$$
\left(Z_{c}\right)^{2}=\left(Z_{T}\right)^{2}+\left(Z_{S}\right)^{2} \beta^{\eta}
$$

where $\tau_{s h}=\sqrt{\left(\tau_{1}\right)^{2}+\left(\tau_{2}\right)^{2}}$ and $\langle x\rangle$ is defined as $\langle x\rangle=\frac{1}{2}(x+|x|)$, and $Z_{T}$ and $Z_{S}$ designate the interlaminar tensile and shear strength, respectively; $\eta$ is a fitting parameter; and $\beta$ is the mode-mixity which is assumed to be given by:

$$
\beta=\frac{\left\langle\tau_{s h}\right\rangle}{\sqrt{\left(\tau_{s h}\right)^{2}+\left(\left\langle\sigma_{3}\right\rangle\right)^{2}}}
$$

The tractions were determined in the plane perpendicular to the interface, such that $\sigma_{3}$ is normal to the interface and $\tau_{2}$ and $\tau_{3}$ are shear tractions. As mentioned previously, the subsequent unloading of the interface elements was performed following the bi-linear mixed-mode traction-separation formulation proposed in [11]. In this formulation, the following relationship needs to be enforced to enable the accurate modeling of large crack growth under mixedmode conditions:

$$
Y_{S}=Y_{T} \sqrt{\frac{G_{I I c}}{G_{I c}}}
$$

where $Y_{\mathrm{T}}$ and $\mathrm{Y}_{\mathrm{S}}$ are material intralaminar tensile and shear strength values.

However, as a consequence, Equation 4 may limit the ability of the approach to accurately capture damage initiation and small crack growth, since the actual material properties may not relate as given in Equation 4 . In DCZ elements, the direction of the crack propagation, $\mathbf{d}$, is estimated and used to affect the elements constitutive response [5] or enhance an onset criterion [10]. In the present work, and following [10], the crack direction $\mathbf{d}$ is assumed to be opposite to the gradient of the displacement jump $\delta_{T}$ determined within the element:

$$
\mathbf{d}=-\nabla \delta_{T}
$$

Contrarily to delamination modeling, where interface elements were pre-assigned along the interface between subelement 1 and 2, matrix cracks were not pre-defined. Under quasi-static loading conditions, matrix crack onset and formation were modeled by splitting the sub-elements along the fiber direction and inserting DCZ elements that open according to their traction-separation formulation. In addition, matrix cracks also onset via the migration. As mentioned previously, once a matrix crack onsets, and a given element is split, a cohesive element is inserted which upon further loading will open according to its traction-separation law. In the present study, the same DCZE formulation described previously is used to model matrix cracks and delaminations. Furthermore, to ensure consistency, the same criterion used to determine the peak of the traction-separation law, Equations 1 and 2, were also used to determine matrix crack onset. This guarantees a smooth transition throughout crack onset, insertion and subsequent opening process. In this case, the tractions were determined in the plane perpendicular to the maximum principal stress obtained in the ' $m n$ ' plane (see Figure 4) transverse to the fibers (hence $\tau_{1}=0$ ). Finally, when assessing Equation 2, interlaminar tensile and shear strengths, $Z_{T}$ and $Z_{S}$, were substituted by intralaminar tensile and shear strengths, $Y_{T}$ and $Y_{S}$, respectively. 


\section{Fatigue:}

In Fatigue, similarly to quasi-static, both delaminations and matrix cracks onset were determined via a Benzeggagh-Kenane (BK) criterion [11] written in stress space. Fatigue crack onset (delamination and matrix crack) is assumed to be approximated by an onset curve with the form [10]:

$$
S=S_{c}(1-\alpha \log (N))
$$

where $S_{c}$ is the static strength, and $\alpha$ a parameter determined experimentally. In the present work, $S_{c}$ was assumed to vary with mode-mixity, following Equation 2, such that:

$$
S_{c}=\left(S_{T}\right)^{2}+\left(S_{S}\right)^{2} \beta^{\eta}
$$

and $S_{T}$ and $S_{S}$ may be replaced by interlaminar or intralaminar static strengths, yielding Equations 1 and 2 . It is important to highlight the there was a lack of experimental data, and accompanying understanding, that can validate this criterion, or any other stress based criterion. This approach was chosen given its simplicity and was directly parallel to what was assumed in modelling damage initiation under quasi-static loading conditions. Fatigue damage accumulation prior to the onset of discrete cracks was obtained by the Palmgren-Miner rule [12]:

$$
\left.\mathrm{C}\right|_{k}=\sum_{s=1}^{k} \frac{\left.\Delta N_{i n c}\right|_{s}}{\left.N_{f}\right|_{s}}
$$

where $\left.N_{f}\right|_{s}$ and $\left.\Delta N_{\text {inc }}\right|_{s}$ are, respectively, the number of cycles to failure and the number of cycles accumulated at the stress corresponding to the $s^{\text {th }}$ step. Equation 8 can also be used to determine the cycles needed to fail an element at a given stress level and step $k$ :

$$
\left.\Delta N_{\text {onset }}\right|_{k}=\left.N_{f}\right|_{k}\left(1.0-\left.C\right|_{k-1}\right)
$$

In the current implementation, $\left.\Delta N_{\text {onset }}\right|_{k}$ was computed for all elements at each increment. At the end of each increment, the minimum number of cycles to onset and/or to propagate a crack was determined and assumed to equal the cycle increment $\left.\Delta N_{i n c}\right|_{s}$. Finally, all elements for which:

$$
\frac{\left.\Delta N_{\text {onset }}\right|_{k}}{\Delta N_{\text {inc }}}<1+f
$$

where $f=0.1$, were considered to fail in step $k$. Once an element fails according to Eq. 10, a matrix crack or delamination of a finite size is inserted. Once a matrix crack is onset, the concept of propagation zone is used, as described in [9], to define a region ahead of the crack tip within which the onset criterion is not activated. This propagation zone was defined by a characteristic length, which also assumed to correspond to the approximate length of the cracks once they are inserted. This enables the subsequent use of VCCT and LEFM assumptions to model growth, combined with a stress-based criterion to determine crack onset.

As mentioned, matrix crack and delamination growth are modeled using a VCCT approach. In the present work, an element-based VCCT is used, following [9]. In this formulation, the shape functions of interface elements were used to obtain tractions and displacements at nodal points. The tractions and displacements were used to compute energy release rates for the elements at the crack front using VCCT. The potential advantage, compared to the classic nodal based VCCT, was the use of the same interface element framework typically used with standard cohesive elements.

Three Paris Law expressions of increasing complexity were considered in the present study. The first assumes that growth rate is directly proportional to $G_{\text {Tmax }}$ :

$$
\frac{\mathrm{d} a}{\mathrm{~d} N}=c(\beta)\left(G_{\text {Tmax }}\right)^{n(\beta)}
$$


where $G_{T \max }$ corresponds to the maximum energy release rate obtained at peak load, $a$ designates the crack length and $N$ the cycles. The coefficient $c(\beta)$ and exponent $n(\beta)$ are assumed, in this study, to be a piece-wise linear function of the mode-mixity. The values for $c$ and $n$ at selected $\beta$ were obtained experimentally, following the normalization procedure outlined in [13].

The dependence of the growth rate on the crack growth increment in tape-laminates has been often observed in unidirectional characterization specimens, and has been typically associated with the development of fiber bridging $[13,14]$. Fiber bridging in these specimens was treated as an artifact, since it was unlikely to occur in the same fashion across interfaces between plies of different orientations. Hence, in order to obtain growth rates that do not incorporate this effect, several approaches can be found in the literature $[13,14]$. The most commonly used approach assumes that growth rates that do not include fiber bridging were obtained via normalization by the static R-curve, $G_{R}(\Delta a)$ [13]. The values for the coefficient, $c(\beta)$, and exponent, $n(\beta)$, used in this study were obtained following this normalization procedure for $\beta<1[13,15]$. However, recent studies have shown that in generic interfaces between plies of different orientations, bridged fibers can also be observed as a result of delamination delving slightly into one of the bounding plies [4]. To assess the sensitivity of the results to this effect, the following expression is assumed:

$$
\left(\frac{\mathrm{d} a}{\mathrm{~d} N}\right)_{\Delta a}=c(\beta)\left(\frac{G_{T \max }}{G_{R}(\Delta a)} G_{C}\right)^{n(\beta)}
$$

where $G_{C}=G_{R}(0)$. This expression is consistent with the normalization procedure used to obtain $c(\beta)$ and $n(\beta)$, mentioned previously. As the crack grows, $G_{\text {Tmax }}$ is multiplied by $\frac{G_{C}}{G_{R}(\Delta a)} \leq 1$, leading to an effective energy release rate that is smaller than $G_{T \max }$, since $G_{R}(\Delta a)$ is typically greater than $G_{C}$ for $\Delta a>0$.

Finally, we also consider an alternative Paris Law formulation in which $G_{\text {Tmax }}$ was replaced by $\Delta \tilde{G}=$ $\left(\sqrt{G_{T \max }}-\sqrt{G_{T \min }}\right)^{2}$. Following [16], it was assumed that this formulation may enable capturing the R-ratio $(R)$ dependence that was reported if the Paris-Law is written in terms of $G_{T \max }$ alone, or as $G_{T \max }-G_{T \min }$. Indeed, substituting $G_{\text {Tmax }}$ by $\Delta \tilde{G}$, Equation 12 can be re-written as a function of the R-ratio and $G_{\text {Tmax }}$

$$
\left(\frac{\mathrm{d} a}{\mathrm{~d} N}\right)_{\Delta a, R}=c_{0}(\beta)(1-R)^{2 n}\left(\frac{G_{T \max }}{G_{R}(\Delta a)} G_{C}\right)^{n(\beta)}
$$

in which $c_{0}(\beta)$ can be related to $c(\beta)$ by:

$$
c_{0}(\beta)=\frac{c(\beta)}{\left(1-R_{0}\right)^{2 n}}
$$

where $R_{0}$ is the R-ratio at which $c(\beta)$ was obtained. One of the novel aspects considered in this work was the calculation of the crack growth increment $\Delta a$ for each crack required to evaluate Eqs 12 and 13. The value of $\Delta a$ for an element $e_{j}$ at the crack front, $\Delta a^{e l_{j}}$ is determined as:

$$
\Delta a^{e l_{j}}=\Delta a^{e l_{G}}{ }_{\max }+a_{l}^{e l_{G \max }}
$$

where $\Delta a^{e l_{G}}{ }_{\text {Tmax }}$ is the crack growth increment for the element $e l_{G_{T \max }}$ in the wake of $e l_{j}$ that is being used to determine $G_{T \max }$ via VCCT, and $a_{l}^{e l_{G_{T \max }}}$ is the length the element $e l_{G_{T \max }}$ along the direction of $e l_{j}$. This implies obtaining and passing information across elements. However, a VCCT implementation already requires interchange of information between elements to compute energy release rates, and thus Eq. 15 can be trivially implemented. 


\section{Migration onset}

Static

Under quasi-static loading, migration onset was determined using the approach outlined in [7]. The first step involves determining the activated ply. The activated ply was the ply towards which, under mixed-mode conditions, micro-cracks forming ahead of the delamination tend to grow [17]. The activated ply was inferred based on the direction of propagation computed by the DCZ elements and the stress state, following the procedure outlined in [7]. If the difference in orientation between the crack direction and the bounding ply was less than a certain assumed value, based on experimental evidence [18], it iwas considered that cracks may indeed initiate and cohesive elements are inserted and will open as described in the previous section. Note that at this point, migration has not completed. Indeed, migration will only complete if energetically favorable.

\section{Fatigue}

The criterion used to predict migration under fatigue shares the same basic premises as the migration criterion used in the quasi-static case follows from [11]. The shear sign and crack propagation direction (determined in this case explicitly via VCCT) were used to determine the activated ply. To assess migration propensity, the mixedmode growth rate calculated at the delamination front was compared to a mode I growth rate. This assumption relies on the experimental observation that the migrated matrix cracks seek a Mode I path as they propagate through-thickness [3]. In a similar fashion to the static case, if this criterion is met and if the mismatch between propagation direction and fiber orientation is less than a critical value, a matrix crack of a finite length throughthickness was inserted using the procedure outlined in the previous section. The delamination was allowed to continue propagating, within the split element, with assumed constant growth rate enabling crack branching. Once delamination traverses the split element, VCCT calculations resume and the growth rate at the delamination front is updated. Once the matrix crack was inserted, despite being represented as a vertical crack, tractions were computed in a rotated plane, and the crack was assumed to propagate under Mode I. Note that migration was not completed at this stage. Migration completion was determined as a result of the relative magnitude of the growth rate of the inserted matrix crack as it developed, and the growth rate of the delamination as it grew past the matrix crack.

\section{Numerical Model and Material Properties}

The boundary conditions were chosen based on the best fit to experimental Digital Image Correlation (DIC) data. Further details on this procedure are given in [1]. Pressure was applied at the clamp region in the first step, followed by displacement applied at a line of nodes along the hinge. The material properties used were summarized in Tables I and II. The $G_{R}(\Delta a)$ values used in the simulation for different mode-mixity $(\beta)$ were obtained based on data from [13, 15] assuming $G_{R}(\Delta a)$ was approximately given by a relatioship of the form:

$$
G_{R}(\Delta a)= \begin{cases}\frac{\left(G_{P L}-G_{c}\right)}{\Delta a_{P L}} \Delta a+G_{P L}, & \Delta a \leq \Delta a_{P L} \\ G_{P L}, & \Delta a>\Delta a_{P L}\end{cases}
$$

where $G_{C}, G_{P L}$ and $\Delta a_{P L}$ are given in TABLE III and the subscript ' $P L$ ' stands for plateau value.

The Paris law coefficients and fatigue onset data used in the simulations are summarized in Tables IV and V respectively.

\begin{tabular}{|c|c|c|c|c|c|c|c|c|}
\hline $\begin{array}{c}E_{11} \\
{[\mathrm{GPa}]}\end{array}$ & $\begin{array}{c}E_{22} \\
{[\mathrm{GPa}]}\end{array}$ & $\begin{array}{c}E_{33} \\
{[\mathrm{GPa}]}\end{array}$ & $\begin{array}{c}G_{12} \\
{[\mathrm{GPa}]}\end{array}$ & $\begin{array}{c}G_{13} \\
{[\mathrm{GPa}]}\end{array}$ & $\begin{array}{c}G_{23} \\
{[\mathrm{GPa}]}\end{array}$ & $v_{12}$ & $v_{13}$ & $v_{23}$ \\
\hline 157.2 & 8.96 & 8.96 & 5.08 & 5.08 & 2.99 & 0.32 & 0.32 & 0.5 \\
\hline
\end{tabular}

TABLE II. STRENGTH AND FRACTURE TOUGHNESS PROPERTIES. INTERLAMINAR AND INTRALAMINAR PROPERTIES ARE ASSUMED TO BE THE SAME.

\begin{tabular}{|c|c|c|c|c|}
\hline $\begin{array}{c}Z_{T}=Y_{T} \\
{[\mathrm{MPa}]}\end{array}$ & $\begin{array}{c}Z_{S}=Y_{S} \\
{[\mathrm{MPa}]}\end{array}$ & $\begin{array}{c}G_{I c} \\
{\left[\mathrm{~N} . \mathrm{mm}^{-1}\right]}\end{array}$ & $\begin{array}{c}G_{I I c} \\
{\left[\mathrm{~N} . \mathrm{mm}^{-1}\right]}\end{array}$ & $\eta$ \\
\hline$\{64[19], 127[20]\}$ & $\{112,223\}$ & $0.24[13]$ & $0.739[21]$ & $2.1[22]$ \\
\hline
\end{tabular}


TABLE III. DELAMINATION RESISTANCE, $G_{R}(\Delta a)$, DEFINITION BASED ON DATA FROM [13, 15$]$.

\begin{tabular}{|c|c|c|c|}
\hline $\begin{array}{c}\text { Mode-mixity, } \\
\beta\end{array}$ & $\begin{array}{c}G_{c} \\
{\left[\mathrm{~N}_{\mathrm{mm}}{ }^{-1}\right]}\end{array}$ & $\begin{array}{c}G_{P L} \\
{\left[\mathrm{~N} . \mathrm{mm}^{-1}\right]}\end{array}$ & $\begin{array}{c}\Delta a_{P L} \\
{[\mathrm{~mm}]}\end{array}$ \\
\hline 0 & 0.24 & 0.381 & 15.24 \\
\hline 0.2 & 0.261 & 0.433 & 17.78 \\
\hline 0.5 & 0.38 & 0.4352 & 3.0226 \\
\hline 0.8 & 0.513 & 0.631 & 5.9944 \\
\hline 1.0 & 0.739 & 0.739 & 0.0 \\
\hline
\end{tabular}

TABLE IV. PARIS LAW COEFFICIENTS FOR MODE I AND MODE II OF IM7/8552 [13, 19].

\begin{tabular}{|c|c|c|}
\hline $\begin{array}{c}\text { Mode-mixity, } \\
\beta\end{array}$ & $\begin{array}{c}c(\beta) \\
\mathrm{mm}-\mathrm{N} / \mathrm{mm}^{2}\end{array}$ & $n(\beta)$ \\
\hline 0 & 73.2111 & 6.710 \\
\hline 0.2 & 2277.1701 & 8.3783 \\
\hline 0.5 & 7.3072 & 5.4486 \\
\hline 0.8 & 4.7860 & 5.1580 \\
\hline 1.0 & 0.2306 & 5.450 \\
\hline
\end{tabular}

TABLE V. FATIGUE ONSET DATA. STATIC STRENGHTS ASSUMED AS GIVEN IN TABLE II.

\begin{tabular}{|c|}
\hline$\alpha$ \\
\hline 0.0766 \\
\hline
\end{tabular}

A typical finite element mesh used for blind-prediction, quasi-static and fatigue, is illustrated in Figure 5. The floating node extended interface element was used to model damage onset and propagation in the center region of the laminate, (Figure 5). The remainder of the specimen was modeled with a combination of solid (C3D8I) and shell (SC8R) Abaqus ${ }^{\circledR}$ elements. Mesh refinement studies were performed for material tensile strengths of both $\mathrm{Y}_{\mathrm{T}}=64$ $\mathrm{MPa}$ and $\mathrm{Y}_{\mathrm{T}}=127 \mathrm{MPa}$, focusing on mesh convergence of the peak load value. This study showed that the peak load predictions obtained by halving the element size, $\mathrm{el}_{\mathrm{x}} \approx 0.04 \mathrm{~mm}$ and $\mathrm{el}_{\mathrm{y}} \approx 0.045$, differed from the predictions obtained with the mesh illustrated in Figure 5 by less than 3\%, the solution was considered to have converged.

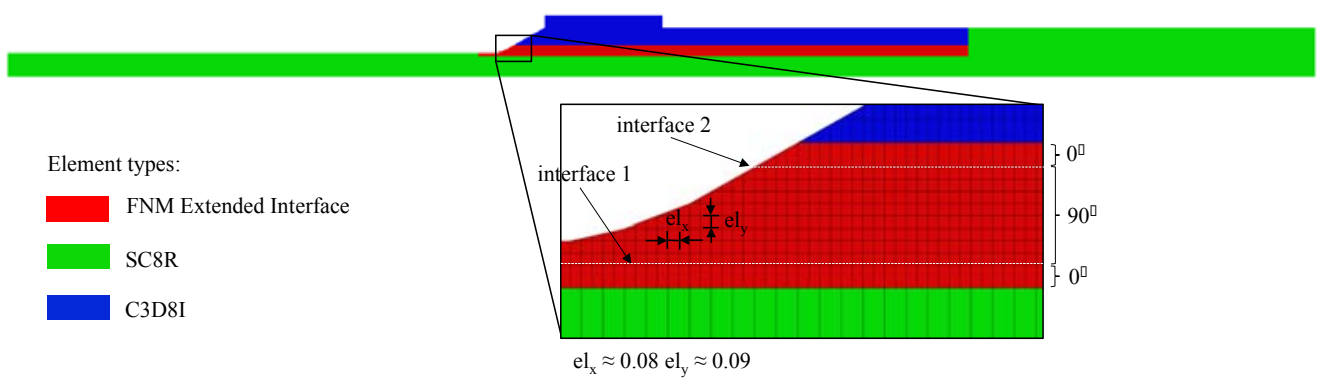

Fig. 5 A typical FE mesh of clamped beam.

\section{B. Quasi-static}

A photograph of a tested specimen, showing the failure morphology, is provided in Figure 6. Both failure morphology and sequence of events followed what was expected, as shown in Figure 2 in Section II. The sequence of events consisted of an initial matrix crack located between interfaces 1 and 2 within the taper region. Subsequently, the matrix crack would transition into a delamination that would grow along "interface 1" and migrate up via a matrix crack to "interface 2". This sequence of events was captured numerically and illustrated in Figure 7. The main difference was that the initial matrix crack location was predicted near interface 2 , at the weak singularity in the taper region, whereas in the experiments it was observed within the taper region between interface 1 and 2. Differences between "as manufactured" vs "as designed" geometry near the taper [7], and potential residual thermal stress effect have been considered as main causes for this discrepancy [23]. In addition, the simulations also predicted multiple migration attempts (vertical red lines) prior to the final migration. Although these could not be observed at the edge of the specimens tested, X-rays of the specimens revealed migration attempts at the center of the specimens. 


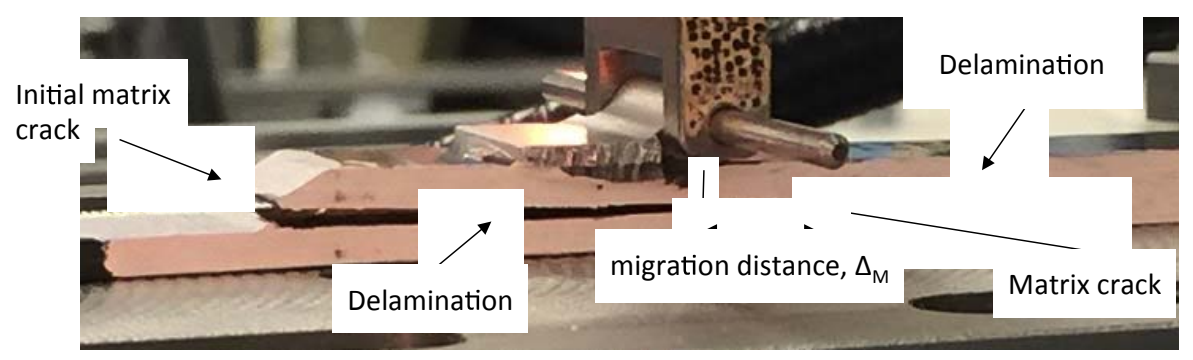

Fig. 6 Final failure morphology of a typical tested specimen.

The blind-predictions for static loading are shown in Figure 8 together with the experimental load-displacement data for load-offset $\mathrm{L}_{\mathrm{A}}$. Two simulations, corresponding to assumed tensile material strengths of $\mathrm{Y}_{\mathrm{T}}=64$ and 127 $\mathrm{MPa}$, are depicted by red and black lines respectively. These two values of $\mathrm{Y}_{\mathrm{T}}$ define lower and upper bounds of material strength values as measured by $90^{\circ}$ tensile or 3-point-bend tests [20,21]. As observed, the simulations showed a strong dependence on the assumed $\mathrm{Y}_{\mathrm{T}}$. In both cases, damage initiation was predicted in the $90^{\circ}$ ply near interface 2 , at the weak singularity.

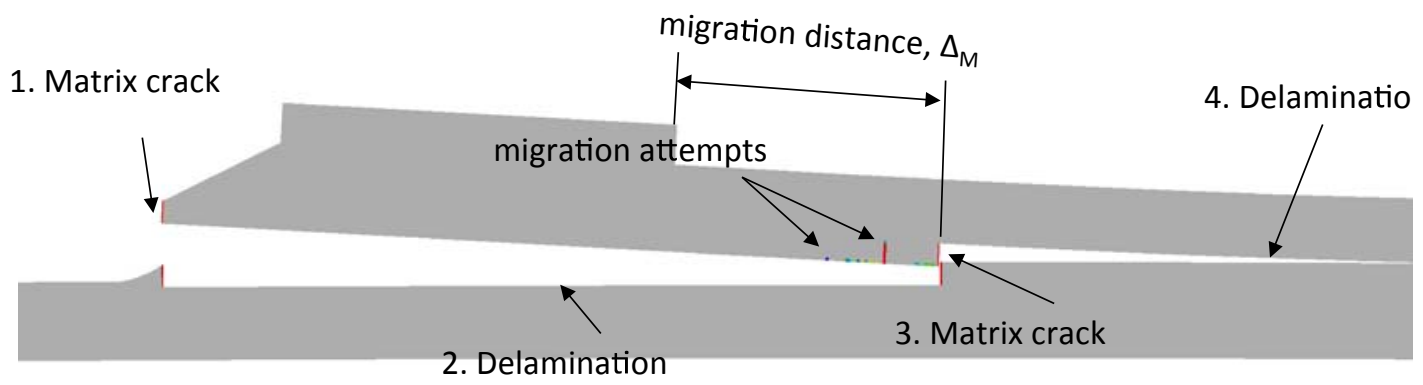

Fig. 7 Final simulated damage state, obtained with $Y_{T}=127 \mathrm{MPa}$.

The migration location predictions under static loading condition were compared with experimental data in Figure 9. Results from blind predictions are shown for two material strength values of $\mathrm{Y}_{\mathrm{T}}=64$ and $127 \mathrm{MPa}$. Migration location was measured from the load application point. Simulations show that migration location occurs at a greater distance from the load application point for $\mathrm{Y}_{\mathrm{T}}=64 \mathrm{MPa}$ compared to the results obtained assuming $\mathrm{Y}_{\mathrm{T}}=127 \mathrm{MPa}$. This difference was attributed to the development of a process zone [7]. Furthermore this difference also illustrates that $\mathrm{Y}_{\mathrm{T}}$ affects not only the peak of the load-displacement, but also the failure morphology. Nevertheless, in both cases the simulation results were within the experimental scatter.

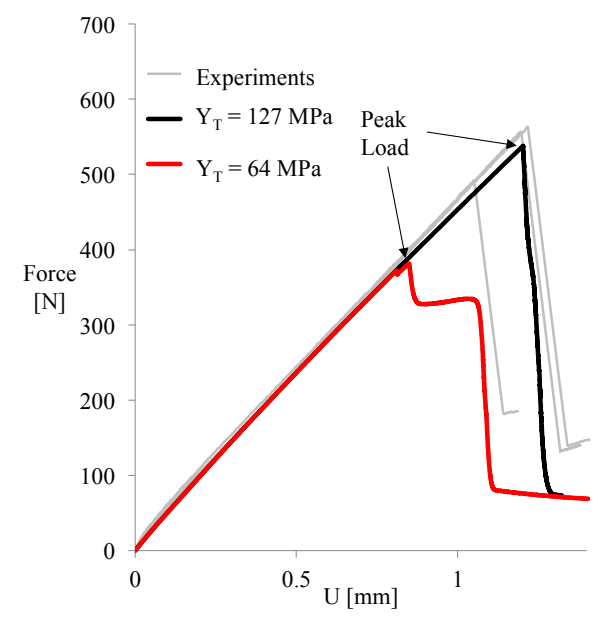

Fig. 8 Comparison of the load-displacement experimental response to simulations obtained with $Y_{T}=64$ and $Y_{T}=127$ MPa. (load application case, $\left.L_{A}\right)$ 


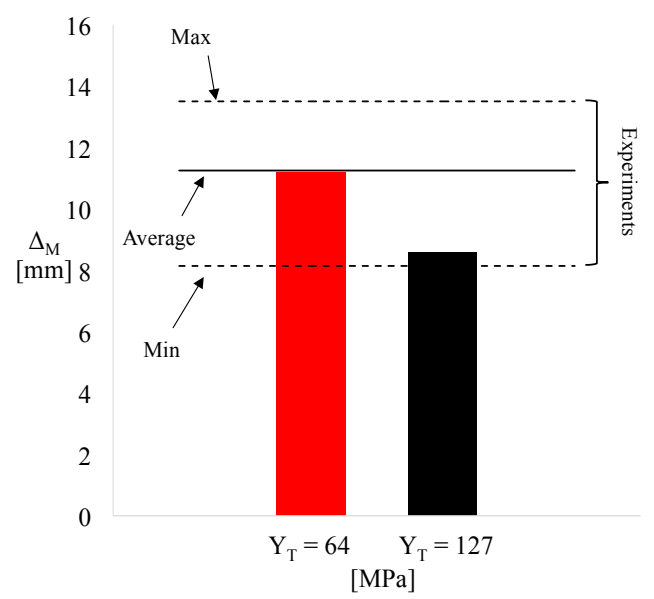

Fig. 9 Comparison of migration location obtained from the experiments and the simulated migration location obtained with $Y_{T}=64$ and $Y_{T}=127 \mathrm{MPa}$. (load application case, $\left.L_{A}\right)$

The comparison of blind-prediction analyses to experimental data for static load-offset location $\mathrm{L}_{\mathrm{B}}$ is shown in Figure 10. Similarly to what was performed for $L_{A}$, two simulations corresponding to assumed tensile material strengths of $\mathrm{Y}_{\mathrm{T}}=64$ and $127 \mathrm{MPa}$ were performed, and depicted by red and black lines, respectively. Simulations showed, as in the $L_{A}$ load case, a strong dependence on $\mathrm{Y}_{\mathrm{T}}$. For both $\mathrm{Y}_{\mathrm{T}}$ values tested, damage initiation was predicted in the $90^{\circ}$ ply near interface 2 , at the weak singularity. The test results in Figure 10 show somewhat different loaddisplacement responses. However, these differences are consistent with differences observed between simulations

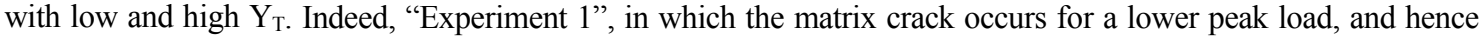
apparent $Y_{\mathrm{T}}$, has a load-displacement shape that resembles the load-displacement curve obtained when assumed the lower $\mathrm{Y}_{\mathrm{T}}$ is assumed, whereas "Experiment 2", where initial damage occurs at a higher load, shows a loaddisplacement curve that follows more closely the simulated load-displacement obtained with the higher $\mathrm{Y}_{\mathrm{T}}$.

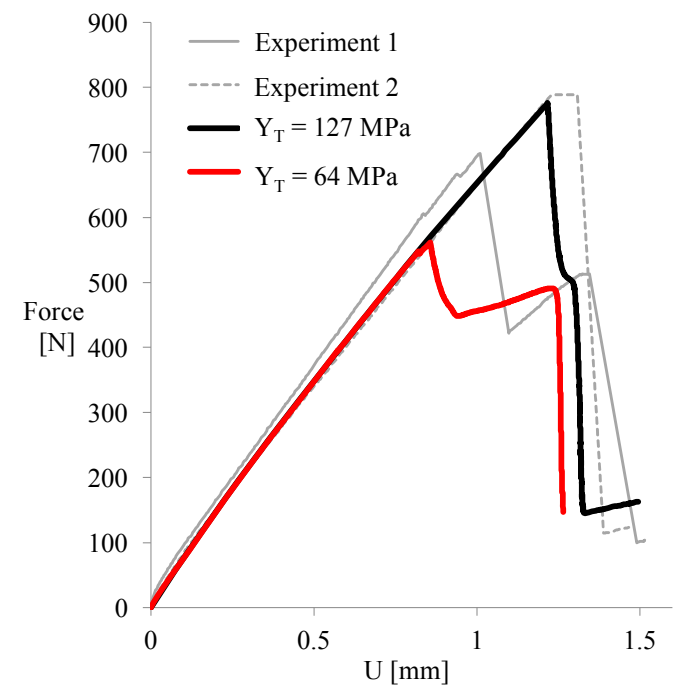

Fig. 10 Comparison of the load-displacement experimental response to simulations obtained with $Y_{\mathrm{T}}=64$ and $\mathbf{Y}_{\mathrm{T}}=127 \mathrm{MPa}$. (load application case, $\left.\mathrm{LB}_{\mathrm{B}}\right)$.

Comparison of final migration location from blind-prediction analyses results, along with experimental data for the second static loading case, $L_{B}$, are shown in Figure 11. Results show that for load case $L_{B}$, migration occurred nearer the loading pin relative to the load case $L_{A}$, and $\Delta_{M}$ was smaller on average. Simulations were able to correctly capture this trend, correlating well with experimental data. Similarly to load case $L_{A}$, the simulations showed dependence of $\Delta_{M}$ on assumed $\mathrm{Y}_{\mathrm{T}}$. These results suggest that $\mathrm{Y}_{\mathrm{T}}$ may not only affect the peak load predictions, but also the matrix crack delamination interaction. Moreover, a low $\mathrm{Y}_{\mathrm{T}}$ value may not always lead to conservative predictions: $\Delta_{M}$ was larger for the smaller $\mathrm{Y}_{\mathrm{T}}$. Furthermore, the commonly used approach of using 
artificially low strengths while keeping the fracture toughness constant, to enable the use of coarser meshes when simulating damage (in particular delamination) with cohesive zone elements, is not recommended in the predictive simulation of any component where crack initiation, small crack growth, and multiple crack interaction may occur.

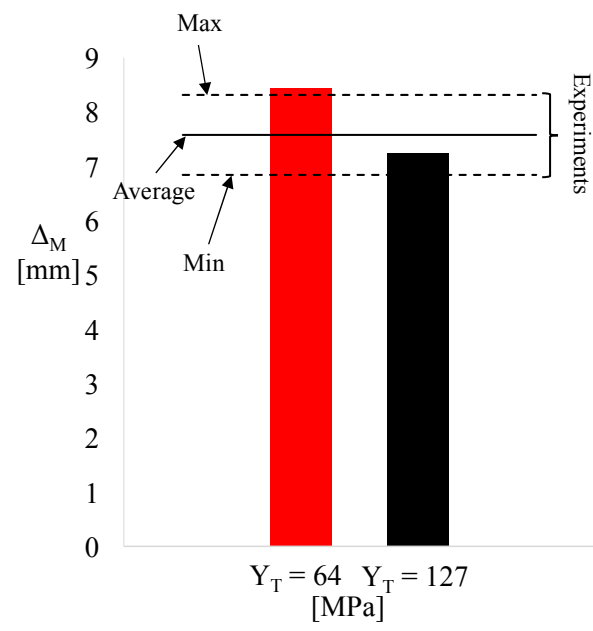

Fig. 11 Comparison of migration location obtained from the experiments and the simulated migration location obtained with $Y_{T}=64$ and $Y_{T}=127 \mathrm{MPa}$. (load application case, $L_{B}$ )

\section{Fatigue}

The blind-predictions made by the FNM code under fatigue loading are detailed in this section. As mentioned in experimental section II, two load levels were considered: low load level corresponding to maximum applied displacement of to $U_{\max }=0.825 \mathrm{~mm}$ and a high load level corresponding to $U_{\max }=0.91 \mathrm{~mm}$. For both load cases, the chosen R-ratio was 0.2 . For each load case, three simulations were performed with different assumed growth laws:

- $(\mathrm{d} a / \mathrm{d} N)$ : Paris Law given by Equation 11, where the coefficient and exponent are obtained by normalizing the experimental data by $G_{R}(\Delta a)$.

- $\quad(\mathrm{d} a / \mathrm{d} N)_{\Delta a}:$ Paris law given by Equation 12 . Assumes there is an active resistance mechanism. Estimates its effect on the crack growth rate by including crack growth increment dependence as measured in characterization tests.

- $\quad(\mathrm{d} a / \mathrm{d} N)_{\Delta a, R}$ : Paris law given by Equation 13. Adds R-ratio dependence to Equation 12 by considering the Paris Law to be a function of $\Delta \tilde{G}$.

Note that the results in Refs. 13 and 15 which were used to generate Eqs. 11 and 12 were determined at $\mathrm{R}=$ 0.1 . Figures 12 and 13 show the results obtained for the extension of the growing delamination (which may be at interface 1 or at interface 2 after migration) as a function of the number of cycles for the two load cases. The results obtained with the growth laws labeled $(\mathrm{d} a / \mathrm{d} N),(\mathrm{d} a / \mathrm{d} N)_{\Delta a}$ and $(\mathrm{da} / \mathrm{dN})_{\Delta a, R}$, as outlined above, are shown in the figures. Overall, the three cases considered were able to bound the experiments and capture a similar growth rate for both low applied load (Figure 12) and high applied load (Figures 13). The main discrepancy pertains to the initiation prediction, which seems to be under-predicted for all cases considered. In addition, the initial matrix crack location was predicted near interface 2, at the weak singularity, in the taper region. In the experiments however, the initial matrix crack occurred between interfaces 1 and 2 within the taper region (see Figure 2). The same possible causes for the similar discrepancy observed under quasi-static loading may be at play in fatigue: differences between "as manufactured" vs "as designed" region in the taper, and the effect of thermal residual stresses. Figures 12 and 13 show that when using Eq. 11, (da/d $N)$ the crack growth predictions are conservative for the specimens tested, whereas, including crack growth increment dependence using Eq. $12,(\mathrm{~d} a / \mathrm{d} N)_{\Delta a}$, seems to provide more realistic results. However, the unidirectional characterization specimens may not yield the representative crack growth increment dependence for interfaces between plies of dissimilar orientation. In addition, Equation 13, $(\mathrm{da} / \mathrm{dN})_{\Delta a, R}$, seems to indicate a non-negligible dependence on R-ratio, 
leading to a slower growth rate, which may contribute to the overly conservative predictions obtained with Eq. 11.

Migration location is overall well predicted, as highlighted in Figures 12 and 13. Furthermore, the simulations seem to indicate a region of slow growth preceding migration, which corresponds to a region associated with multiple migration attempts. Such a region can also be found in the experiments, and was particularly noticeable in the low load case, Figure 12. While migration attempts were found in the experiments prior to migration, further investigation was needed to assess whether they were the main contributor for the slower rate observed.

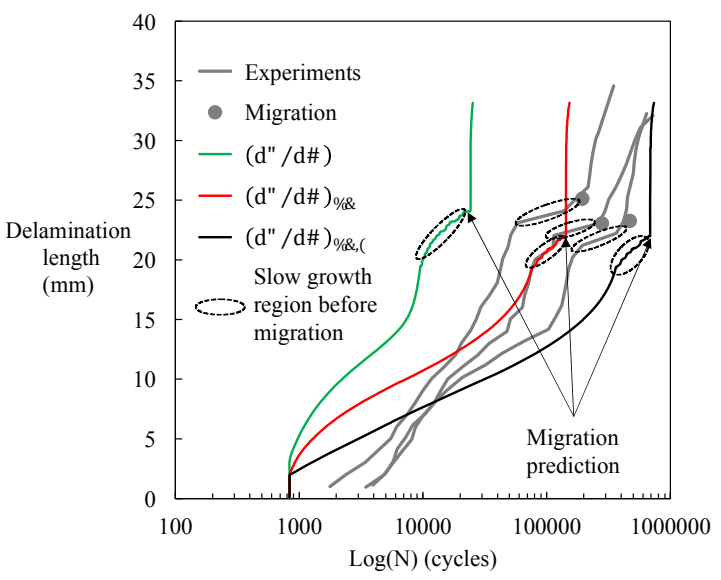

(a) $\log$ plot

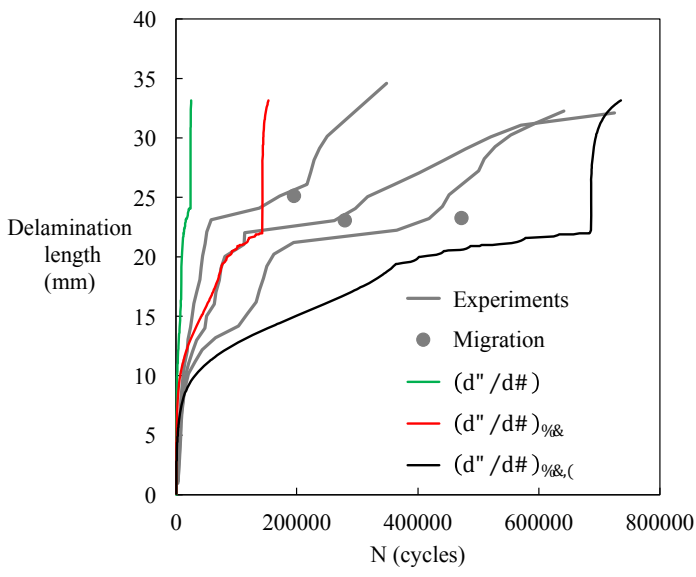

(b) linear scale

Fig. 12 Comparison of number of cycles with delamination extension, low load level.

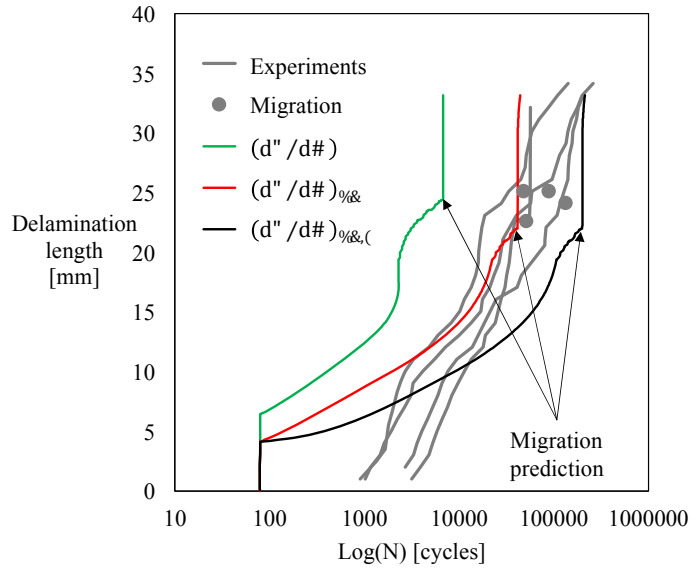

(a) $\log$ plot

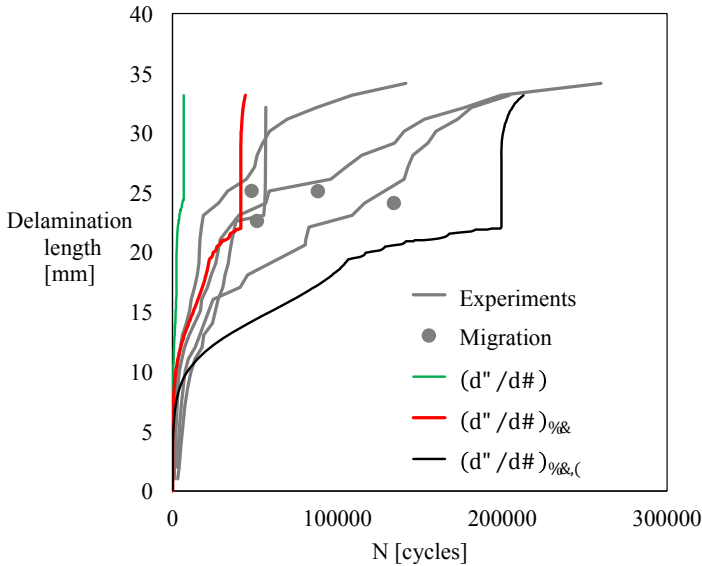

(b) linear scale

Fig. 13 Comparison of number of cycles with delamination extension, high load level.

\section{Concluding Remarks}

The FNM (Floating Node Method) was combined with DCZE and VCCT to model the clamped taper beam specimen under quasi-static and fatigue loading. Delamination, matrix cracking, and migration were all modeled with the same FNM element.

The simulations were able to correctly predict the sequence of events and failure morphology under both quasistatic and fatigue loading conditions. The main discrepancy observed was in the prediction of the initial matrix 
crack location. This discrepancy may be caused by the difference between "as manufactured" vs "as designed" specimen geometry near the matrix crack initiation site, and the possible effect of residual thermal stresses.

The quasi-static load-displacement predictions agreed well with the experimental results when the high $\mathrm{Y}_{\mathrm{T}}$ value was used. Predictions obtained assuming the low $\mathrm{Y}_{\mathrm{T}}$ under-predicted the peak of the load-displacement response. Similar agreement was obtained for the two load offsets tested, $L_{A}$ and $L_{B}$. Migration location was overall well predicted for the two load cases, however, it also showed dependence on $\mathrm{Y}_{\mathrm{T}}$. Results indicate that to increase the predictive capability, further investigation may be required on the characterization and use of $\mathrm{Y}_{\mathrm{T}}$ to predict initial matrix crack formation.

Fatigue simulation results, for both load cases considered, bounded the experimental results obtained, except near onset. Overall, results showed good agreement including the qualitative prediction of regions of slower/faster growth rate and migration location. The predictions obtained with the mixed-mode Paris Law formulation, that did not consider growth rate as function of the crack growth increment, were always overly conservative. On the other hand, including the crack growth increment and R-ratio dependence in the Paris Law formulations provided growth predictions that tended to be less conservative and agree better with the experimental results. However, it is worth mentioning that the unidirectional characterization specimens used to define the growth laws assumed in this study may not yield the representative crack growth increment dependence for interfaces between plies of dissimilar orientation. In addition, the R-ratio dependence of this material system has not yet been thoroughly assessed. Hence, further investigation of this these two effects, R-ratio and crack growth increment dependency, is considered key to improve and further assess the predictive capability of the methodology.

\section{Acknowledgements}

The authors wish to thank the National Aeronautics and Space Administration (NASA) for providing funding for this work under Contract Number: NNL09AA00A.

\section{References}

1. Chen, B.Y., S.T. Pinho, N.V. De Carvalho, P.M. Baiz, and T.E. Tay. "A Floating Node Method for the Modelling of Discontinuities in Composites," Engineering Fracture Mechanics, 27:104-134, 2014.

2. Ratcliffe, J.G., Deobald, L., Mabson, G. and N.V. De Carvalho. "A Clamped Tapered Beam Test to Study Matrix Crack Initiation and Delamination Migration in a Cross-ply Tape Laminate," NASA-TM-2017-219623, 2017.

3. Ratcliffe, J.G., Czabaj, M.W. and T.K. O'Brien. "A Test for Characterizing Delamination Migration in Carbon/Epoxy Tape Laminates,” NASA/TM-2013-218028, 2013.

4. Ratcliffe, J.G. and N.V. De Carvalho. "Investigating Delamination Migration in Composite Tape Laminates," NASA/TM-2014-218289, 2014.

5. Saether, E. and E. Glaessgen. "The Development of Directional Decohesion Finite Elements for Multiscale Failure Analysis of Metallic Polycrystals," NASA/TM-2009-215715, 2009.

6. De Carvalho, N. V. and R. Krueger. "Modeling Fatigue Damage Onset and Progression in Composites Using an Element-Based Virtual Crack Closure Technique Combined with the Floating Node Method," $31^{\text {st }}$ Technical Conference, Williamsburg, VA, USA, 2016.

7. De Carvalho, N.V., Seshadri, B.R., Ratcliffe, J. G., Mabson, G.E. and Deobald, L.R. "Simulating matrix crack and delamination interaction in a clamped tapered beam," 32nd ASC Technical Conference, West Lafayette, IN, USA, 2017.

8. De Carvalho, N.V., Ratcliffe, J.G., Chen, B.Y., Pinho, S.T., Baiz, P.M. and Tay, T.E. "Modeling quasi-static and fatigue-driven delamination migration," $29^{\text {th }}$ ASC Technical Conference, La Jolla, CA, USA, 2014.

9. Kawashita, L.F., Pernice, M.F. and S.R. Hallett. "Mesh-Independent Cohesive Crack Model for the Analysis of Transverse Matrix Cracks in Laminated Composites," presented at $I V$ ECCOMAS Thematic Conference on the Mechanical Response of Composites, 25 - 27 September, 2013.

10. Steenstra, W., van der Meer, F.P. and L.J. Sluys. "An efficient approach to the modeling of compressive transverse cracking in composite laminates," Composite Structures, 128:115-121, 2015.

11. Turon, A., Camanho, P.P., Costa, J. and J. Renart. "Accurate simulation of delamination growth under mixed-mode loading using cohesive elements: Definition of interlaminar strengths and elastic stiffness," Composite Structures, 92(8):1857-1864, 2010.

12. Miner, M.A. "Cumulative Damage in Fatigue," Journal of Applied Mechanics, 67:A159-A164, 1945.

13. Murri, G.B. "Evaluation of Delamination Onset and Growth Characterization Methods under Mode I Fatigue Loading," NASA/TM-2013-21766, 2013.

14. De Carvalho, N. V. and G. Murri. "A Novel Method for Characterizing Fatigue Delamination Growth Under Mode I Using the Double Cantilever Beam Specimen," $29^{\text {th }}$ ASC Technical Conference, La Jolla, CA, USA, 2014. 
15. Ratcliffe, J.G. and W. Johnston. "Influence of Mixed Mode I-Mode II Loading on Fatigue Delamination Growth Characteristics of a Graphite Epoxy Tape Laminate," 29 $9^{\text {th }}$ ASC Technical Conference, La Jolla, CA, USA, 2014.

16. Rans, C., R. Alderliesten, Rinze Benedictus. "Misinterpreting the results: How similitude can improve our understanding of fatigue delamination growth," Composites Science and Technology, Volume 71, Issue 2, Pages 230-238, 2011.

17. Greenhalgh, E. S., C. Rogers, and P. Robinson. "Fractographic observations on delamination growth and the subsequent migration through the laminate," Composites Science and Technology, Volume 69, Issue 14, Pages 2345 2351, 2009.

18. Czabaj, M.W., Davidson, B.D. and J.G. Ratcliffe. "A Modified Edge Crack Torsion Test for Measurement of Mode III Fracture Toughness of Laminated Tape Composites," $31^{\text {st }}$ ASC Technical Conference, Williamsburg, VA, USA, 2016.

19. Makeev, A., Nikishkov, Y., Seon, G. and E. Lee. "Analysis Methods Improving Confidence in Material Qualification for Laminated Composites," Proceedings of the AHS 72nd Annual Forum, May 17-19, 2016, USA, 2016.

20. NIAR Report: NCP-RP-2009-028 Revision A., 2009.

21. O'Brien, T.K., Chawan, A.D., DeMArco, K. and I. Paris. "Influence of Specimen Preparation and Specimen Size on Composite Transverse Tensile Strength and Scatter," NASA/TM-2001-211030, 2001.

22. O’Brien, T.K., Johnston W.M., and G.J. Toland. "Mode II Interlaminar Fracture Toughness and Fatigue Characterization of a Graphite Epoxy Composite Material,” NASA/TM-2010-216838, 2010.

23. Adluru, H.K., Hoos, K.H., and J.I. Endel. "Discrete Damage Modelling of Delamination Migration in Clamped Tapered Laminated Beam Specimens,” 32nd ASC Technical Conference. West Lafayette, IN, USA, 2017. 